\title{
ECONOMÍA MUNDIAL: RECUPERACIÓN ECONÓMICA LENTA DEBIDO A LA PERSISTENCIA DE RIESGOS
}

\author{
GLOBAL ECONOMY: SLOW ECONOMIC RECOVERY \\ RISK DUE TO THE PERSISTENCE \\ BeAtriz Herrra García* \\ Docente Principal de la Facultad de Ciencias Contables \\ Universidad Nacional Mayor de San Marcos-UNMSM / Lima-Perú \\ [Recepción: Julio de 2013/ Conformidad: Agosto 2013]
}

\section{RESUMEN}

Un empeoramiento de la crisis de la zona euro, el abismo fiscal en los Estados Unidos y una fuerte desaceleración de China pueden en conjunto generar una nueva recesión mundial. La tercera posibilidad, una brusca desaceleración de la economía china es significativa, debido a que sus exportaciones continuaron reduciendo su ritmo de crecimiento durante el 2012, debido a la débil demanda en las mayores economías desarrolladas. El crecimiento de la inversión que contribuyó en más del 50 por ciento del PIB chino en las últimas décadas, se ha desacelerado. Debido a ello, existen importantes riesgos de un crecimiento significativamente menor. Si su crecimiento se redujera en cerca del 5 por ciento anual (causado por una mayor desaceleración de la inversión, mayores restricciones al mercado de la vivienda y ausencia de nuevos estímulos fiscales), los países en desarrollo en conjunto podrían sufrir una pérdida acumulada del PIB de cerca del 3 por ciento durante 2013-2015, y el mundo una pérdida del producto mundial del 1.5 por ciento.

\section{Palabras Claves:}

Crisis, crecimiento, riesgos e incertidumbre, economía mundial.

\begin{abstract}
A worsening of the euro zone crisis, the fiscal cliff in the United States and a sharp slowdown in China can together generate a new global recession. The third possibility, a sharp slowdown in the Chinese economy is significant, because its exports continued to reduce its rate of growth during 2012, due to weak demand in major developed economies. The investment growth contributed more than 50 percent of China's GDP in recent decades, has slowed. As a result there are significant risks significantly lower growth. If their growth is reduced by about 5 percent per year (due to a greater slowdown in investment, greater restrictions on the housing market and the absence of further fiscal stimulus), developing countries as a whole could suffer a cumulative loss of GDP of about 3 percent during 2013-2015, and the world global output loss of 1.5 percent.
\end{abstract}

\section{Keywords:}

Crisis, growth, risk and uncertainty, global economy.

* Doctora en Ciencias Económicas. Economista. Docente investigadora. UNMSM. Email: bherrerag@unmsm.edu.pe 


\section{INTRODUCCIÓN}

De acuerdo a los informes económicos emitidos por las Naciones Unidas (UNDESA), existen riesgos evidentes de que el escenario económico mundial pudiera empeorar, y que por tanto, las estimaciones asumidas en el pronóstico base (véase Cuadro 1), no se cumplan y tengan que ser reajustadas a la baja. Según UNDESA el fracaso de los formuladores de políticas en Europa y en los Estados Unidos, para enfrentar la crisis de empleo y prevenir la escalada de problemas asociados a las deudas soberanas y la fragilidad del sector financiero, son el sustento de una base para un agudo riesgo que enfrenta el panorama económico mundial para el 2013 y se prevé una recuperación económica lenta debido a la persistencia de dichos riesgos.
En el Cuadro 1, se puede apreciar cómo la economía mundial se debilitó considerablemente durante 2012. Los países de la zona euro cayeron ese año nuevamente en recesión (-0.5), mientras otros países europeos agobiados por los problemas de su deuda soberana, la han profundizado. El panorama es que muchos países desarrollados se encuentran atrapados en una espiral negativa caracterizada por el alto desempleo y la débil demanda agregada, en medio de austeridad fiscal, elevada carga de deuda soberana y fragilidad financiera. En el año 2012, la economía mundial retrocedió 0.5 por ciento en relación al año anterior ( $2.7 \%$ en 2011 y $2.2 \%$ en 2012 ), estimándose según los pronósticos de Enero del 2013 (UNDESA), una débil recuperación para los dos años siguientes.

\section{Cuadro $\mathrm{N}^{\circ} 1$ \\ Crecimiento del Producto Mundial, 2010-2014 \\ Variaciones porcentuales}

\begin{tabular}{|c|c|c|c|c|c|}
\hline & 2010 & 2011 & 2012 & $2013^{*}$ & $2014^{*}$ \\
\hline Mundo & 4.0 & 2.7 & 2.2 & 2.4 & 3.2 \\
\hline Economías Desarrolladas & 2.6 & 1.4 & 1.1 & 1.1 & 2.0 \\
\hline Estados Unidos & 2.4 & 1.8 & 2.1 & 1.7 & 2.7 \\
\hline Japón & 4.5 & -0.7 & 1.5 & 0.6 & 0.8 \\
\hline Euro Zona & 2.1 & 1.5 & -0.5 & 0.3 & 1.4 \\
\hline Economías en Desarrollo & 7.7 & 5.7 & 4.7 & 5.1 & 5.6 \\
\hline Sudeste Asiático & 9.0 & 6.8 & 5.5 & 6.0 & 6.3 \\
\hline China & 10.3 & 9.2 & 7.7 & 7.9 & 8.0 \\
\hline India & 9.6 & 6.9 & 5.5 & 6.1 & 6.5 \\
\hline América Latina & 6.0 & 4.3 & 3.1 & 3.9 & 4.4 \\
\hline
\end{tabular}

Fuente: United Nations, World Economic Situation and Prospects 2013.

* Datos pronosticados 2013-2014

Sin embargo, el año pasado el comportamiento de las economías en desarrollo jugaron un importante rol en la dinámica de la economía mundial, especialmente las economías del sudeste asiático encabezadas por China (7.7\%) e India (5.5\%), que contrarrestaron el mal desempeño de las economías desarrolladas.
Durante el primer semestre de 2013, se observó un tenue crecimiento de la economía global lo que motivó que se realizaran nuevas proyecciones en Junio de 2013, los resultados se observan en el cuadro siguiente. 


\section{Cuadro $\mathbf{N}^{\circ} 2$}

Pronósticos Revisados del Crecimiento de la Producción Mundial. 2011-2014 Variaciones porcentuales

\begin{tabular}{|c|c|c|c|c|c|c|}
\hline \multicolumn{9}{|c|}{} & \multicolumn{2}{c|}{$\begin{array}{c}\text { Desviación respecto al } \\
\text { pronóstico de } \\
\text { Enero 2013 }\end{array}$} \\
\hline Mundo & 2011 & 2012 & $2013^{*}$ & $2014^{*}$ & $2013^{*}$ & $2014^{*}$ \\
\hline Economías Desarrolladas & 2.8 & 2.3 & 2.3 & 3.1 & -0.1 & -0.1 \\
\hline Estados Unidos & 1.4 & 1.2 & 1.0 & 2.0 & -0.1 & 0.0 \\
\hline Japón & 1.8 & 2.2 & 1.9 & 2.6 & 0.2 & -0.1 \\
\hline Zona Euro & -0.6 & 2.0 & 1.3 & 1.6 & 0.7 & 0.8 \\
\hline Economías en Desarrollo & 1.4 & -0.6 & -0.4 & 1.1 & -0.7 & -0.3 \\
\hline Sudeste Asiático & 5.8 & 4.6 & 5.0 & 5.4 & -0.1 & -0.2 \\
\hline China & 6.9 & 5.5 & 5.8 & 6.1 & -0.2 & -0.2 \\
\hline India & 9.2 & 7.8 & 7.8 & 7.7 & -0.1 & -0.3 \\
\hline América Latina & 7,5 & 5.1 & 5.5 & 6.1 & -0.6 & -0.4 \\
\hline Sud América & 4.3 & 3.0 & 3.6 & 4.2 & -0.3 & -0.2 \\
\hline
\end{tabular}

Fuente: United Nations, World Economic Situation and Prospects as of mid-2013.

* Pronóstico a mitad de año 2013

El Cuadro 2, muestra las posibles implicaciones de un escenario más pesimista. El PIB de la zona euro caería en - 0.4 por ciento en 2013, mientras que Estados Unidos lo haría en un 1.9 por ciento, 0.2 mayor que el pronóstico anterior (véase, desviación). Debido a la situación económica mundial y las perspectivas a mediados de 2013, las economías en desarrollo presentan una menor dinámica configurada en las desviaciones calculadas; sin embargo, su comportamiento sigue siendo fundamental para el desempeño de la economía mundial.

Las economías de China, India y Sudamérica han visto reducido sus tasas de crecimiento en función del tenue crecimiento global en 2013 y cierta mejora esperada en 2014. A pesar de las mejoras de las condiciones financieras globales y la reducción de riesgos a corto plazo (no así a los riesgos de mediano plazo), la economía mundial presenta un cuadro de expansión a un ritmo de débil a moderado.

Después de un descenso marcado en los dos últimos años (la economía mundial creció $4.0 \%$ en 2010, véase, Cuadro 1), la actividad económica mun- dial espera ganar poco a poco impulso en el segundo semestre de 2013. En la mayoría de las regiones del mundo, sin embargo, el crecimiento de mantendrá por debajo del potencial.

En el escenario base (Cuadro 1), el crecimiento mundial se ha revisado ligeramente a la baja, así las previsiones presentadas en el Cuadro 3 , indican que el crecimiento del producto bruto mundial se proyecta en un 2.3 por ciento en 2013, al mismo ritmo de crecimiento del 2012, mientras se espera una recuperación gradual hasta un 3.1 por ciento en 2014. Esto debido a la vigencia de varios riesgos e incertidumbres claves, especialmente de mediano plazo, y si no se mitigan podrían debilitar los esfuerzos para encarrilar al crecimiento mundial.

Finalmente, obsérvese en el Cuadro 3, la desaceleración en el crecimiento esperado para 2013, en los países de la Zona Euro, América Latina, Sudamérica e India es superior a la desaceleración esperada (respecto al escenario base) para los demás países y regiones del mundo. Lo que significa que varias regiones (y subregiones y países), podrían sufrir caídas en sus 
niveles de ingreso per cápita, causando problemas y retrocesos en la lucha por reducir la pobreza.

\section{MODERACIÓN EN EL RITMO DE CRECI- MIENTO DEL COMERCIO MUNDIAL}

Las economías en desarrollo probablemente recibirán significativos impactos negativos en este escenario más pesimista. Los impactos variarán en dependencia de los vínculos económicos, comerciales y financieros que ellos mantienen con las principales economías desarrolladas. Así, se estima que las economías del Sudeste Asiático sufrirán principalmente por las caídas en sus exportaciones a las economías industrializadas, mientras que los países de América Latina, África y Asia Occidental se verían afectadas por la aguda declinación esperada de los precios internacionales de las materias primas. Todas las regiones en desarrollo tendrán que hacer frente a shocks financieros, incluyendo los efectos de contagio en sus mercados de valores, la reversión de los flujos de capital y pérdidas financieras directas como producto de la caída en el valor de los bonos soberanos de las economías desarrolladas.

El crecimiento del comercio mundial se ha desacelerado notablemente por segundo año consecutivo, cayendo desde un crecimiento del 6.9 por ciento en 2011 a un 2.7 por ciento en 2012, véase Cuadro 3.

\section{Cuadro $\mathrm{N}^{\circ} 3$}

Comercio Mundial de Bienes y Servicios, 2011-2014

(Variaciones porcentuales)

\begin{tabular}{|c|c|c|c|c|c|c|}
\hline \multicolumn{4}{|c|}{} & \multicolumn{2}{|c|}{$\begin{array}{c}\text { Desviación } \\
\text { respecto al } \\
\text { pronóstico de } \\
\text { Enero 2013 }\end{array}$} \\
\hline \multicolumn{2}{|c|}{} \\
\hline Comercio Mundial & 2011 & 2012 & 2013 & 2014 & 2013 & 2014 \\
\hline Producto Mundial & 6.9 & 2.7 & 3.5 & 5.0 & -0.8 & 0.1 \\
\hline
\end{tabular}

Fuente: United Nations, World Economic Situation and Prospects as of mid-2013.

El débil crecimiento económico mundial, especialmente en Europa y otras economías desarrolladas, es el principal factor determinante de esta desaceleración. En el Cuadro 3 se observa que se ha desacelerado notablemente en 2012 (2.7\%), es una fuerte caída desde el crecimiento del volumen de las exportaciones mundiales de bienes a una tasa de $12.8^{1}$ por ciento en 2010. Para el 2013, la proyección en el escenario base es que el crecimiento del comercio mundial será moderado (3.5\%) para luego volver cerca de su tasa de crecimiento promedio de largo plazo, en torno al 5 por ciento en 2014.

En ese escenario los países en desarrollo se han mostrado más resistentes a la nueva desaceleración debido a su importancia en el comercio mundial. Su participación continúa aumentando, en concordancia

${ }^{1}$ Cuadro $\mathrm{N}^{\circ} 3.1$

DINAMISMO DEL COMERCIO MUNDIAL DE BIENES Y SERVICIOS, 2010-2011

(Volumen en porcentajes)

\begin{tabular}{|c|c|c|}
\hline & 2010 & 2011 \\
\hline 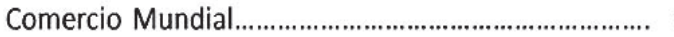 & 12.8 & 6.9 \\
\hline 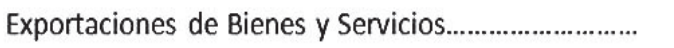 & 11.5 & 5.7 \\
\hline Importaciones de Bienes y Servicios.... & 11.3 & 4.8 \\
\hline
\end{tabular}

Fuente: Economic Outlook, OECD, París, 2012

64/ QVIPUKAMAYOC | Vol. 21(40) 2013 
con su integración a las cadenas globales de valor. Durante la crisis se espera se mantenga la dicotomía entre un robusto crecimiento del comercio exterior de bienes en las economías en desarrollo, por una parte, y un débil comportamiento en las transacciones externas de los países desarrollados, por la otra.

Durante la primera mitad de 2012 los precios de las materias primas en los mercados mundiales se mantuvieron con una fuerte alza. Sin embargo, en la segunda mitad, los precios de la mayoría de los productos básicos se redujeron moderadamente, debido a percepciones infundadas de una rápida recuperación de la economía mundial. Los precios de los alimentos y combustibles se mantuvieron elevados durante el tercer trimestre de 2012, debido, principalmente a las condiciones climáticas adversas en varios países. Los precios de los minerales y metales continuaron deprimiéndose ante el sombrío panorama económico mundial. En los próximos años, los exportadores de materias primas, que se han beneficiado de mejores términos de intercambio en los últimos años, continuarán expuestos a presiones hacia la baja.

La fuerte recuperación del comercio de servicios experimentada en todas las regiones debido a los sectores de transporte de carga, intensivos en emisiones de carbono, y de viajes se ha mantenido en expansión en los países en desarrollo. Se espera que los servicios de transporte de carga continúen en expansión en consonancia con la expansión del comercio a través de las cadenas globales de valor.

Los factores que afectan las tendencias de los precios de las materias primas son: a) una perspectiva menos sombría respecto al dinamismo de la economía mundial; b) aumentos perceptibles en los niveles de oferta en algunos mercados; c) las liquidaciones de contratos de commodities en los mercados derivados financieros que ocurrieron simultáneamente con la caída en los mercados financieros globales; y, d) una perceptible apreciación del dólar estadounidense. En perspectiva, se estima que los precios internacionales de la mayoría de los productores primarios continuarán moderándose en 2013, como ya se observó durante la segunda mitad de 2012, dada la debilidad del crecimiento económico mundial y del comercio global.

\section{RIESGOS E INCERTIDUMBRES LATENTES}

El empeoramiento de la crisis en la zona euro está generando más dificultades. La actual dinámica, compuesta por la crisis de la deuda soberana y la fragilidad del sector bancario, está afectando negativamente las hojas de balance tanto de los gobiernos como de los bancos comerciales. La respuesta aplicada por los gobiernos, austeridad fiscal, está exacerbando la desaceleración económica, creando efectos adversos sobre el mismo objetivo de consolidación fiscal y elevando los ratios de deuda, lo que provoca nuevos recortes presupuestarios.

El abismo fiscal en los Estados Unidos, estima que este país podría caer en el llamado abismo fiscal; es decir, un estancamiento político podría complicar un nuevo acuerdo presupuestario, lo que podría en acción reducciones de gastos, incluyendo una reducción en los gastos públicos en un cifra estimada cercana a los cien mil millones de dólares, y un aumento de impuestos por cerca de la mitad de la anterior cifra. Según expertos de Naciones Unidas, con ese programa automático de austeridad fiscal durante el período 2013-2015 Estados Unidos estaría en torno al 4.0 por ciento del PIB; dichos expertos afirman que es ese escenario de abismo fiscal, el crecimiento económico mundial se reduciría a la mitad en 2013, mientras que para el 2015 se reduciría en 2,5 por ciento.

Una fuerte desaceleración de la economía China y de otros principales países en desarrollo es una posibilidad. De hecho el crecimiento económico se redujo considerablemente durante 2012 para varias de las mayores economías en desarrollo como China, India y Brasil, países que registraron períodos de fuerte crecimiento previo a la crisis financiera y que pudieron recuperarse rápidamente en 2010, luego de la gran recesión. Debido a las incertidumbres existentes en la demanda externa y los diferentes desafíos internos que enfrentan estos países, los riesgos de una mayor desaceleración en el crecimiento en estas economías son significativos. 
En China las exportaciones continúan reduciendo su ritmo de crecimiento, debido a la débil demanda de las mayores economías industrializadas. El crecimiento de la inversión que contribuyó en más del 50 por ciento al crecimiento de la economía china en las últimas décadas, se ha desacelerado a partir de 2012. Las razones que explican este comportamiento son las políticas más restrictivas en el mercado de la vivienda, mayor precaución en las medidas de estímulo fiscal y restricciones de financiamiento que han enfrentado los gobiernos locales en la implementación de nuevos proyectos. Debido a estos factores, existen importantes riesgos de un crecimiento significativamente menor de la economía china. Sí el crecimiento de la economía china se redujera, causado por una mayor desaceleración de la inversión, mayores restricciones al mercado de la vivienda y ausencia de nuevos estímulos fiscales, a cerca del 5.0 por ciento anual los países en desarrollo, en conjunto, podrían sufrir una pérdida acumulada del producto en cerca del 3.0 por ciento durante los próximos tres años.

\section{DESACELERACIÓN EN LAS ENTRADAS DE INVERSIÓN EXTRANJERA DIRECTA (IED) HACIA LOS PAÍSES EN DESARROLLO}

Las entradas netas de capital privado hacia los países en desarrollo continuaron recuperándose luego de la abrupta caída experimentada durante la crisis de 2009, pero sufrieron un fuerte retroceso a partir del tercer trimestre de 2011, con el marcado deterioro que experimentaron los mercados financieros internacionales. Las previsiones respecto al financiamiento externo están sujetas a las incertidumbres derivadas de dos fuerzas contradictorias que vienen operando.
Por una parte, la continuidad de los problemas de la deuda soberana en países desarrollados, y la correspondiente volatilidad en los mercados de capital, lo cual reduciría las inversiones de portafolio en las economías en desarrollo. Es decir, una profundización de la crisis de la deuda soberana pudiera conducir a desapalancamientos de capital en las instituciones financieras de los países desarrollados o en la fuga hacia inversiones seguras (en activos denominados en dólares o en francos suizos, como ocurrió en el tercer trimestre de 2011).

Por otra parte, las perspectivas de crecimiento en las principales economías en desarrollo las hará más atractivas para recibir flujos de inversión extranjera directa (IED), mientras que los diferenciales de tasas de interés continuarían favoreciendo el financiamiento en estos países, a pesar de que las primas de riesgo para algunos de ellos aumenten ${ }^{2}$.

El aumento hacia la aversión al riesgo ha reducido recientemente los flujos de capital hacia las economías en desarrollo, aunque las emisiones de deuda en monedas locales de estos países han continuado atrayendo flujos de capital desde la zona euro en crisis. Los spreads (sobrecostos) de deuda soberana y corporativa de los países desarrollados siguen en alza, mientras que los bancos de los países en desarrollo han estado incrementando las exigencias para el otorgamiento de financiamiento en un escenario de crecientes préstamos no rembolsables y condiciones agravadas de financiamiento.

Sin embargo, los indicadores de demanda crediticia son aún positivos en todas las regiones, y de hecho el crecimiento del crédito total (interno y externo) ha caído desde sus niveles más altos, pero se mantienen elevados en muchas economías en desarrollo.

\footnotetext{
2 Aunque las perspectivas son contradictorias según el Banco Mundial, se espera en términos relativos, como proporción del PIB, una desaceleración en las estradas de flujos de capital privado (IED) hacia las distintas regiones en desarrollo para los años 2012, 2013 y 2014. En esa misma línea el FMI informa que los préstamos bancarios se mantienen estancados en las economías desarrolladas, y la confianza en el sistema financiero global permanece excepcionalmente frágil.
} 


\section{Cuadro $\mathrm{N}^{\circ} 4$}

Flujos Netos de Capitales hacia los Países en Desarrollo

(En miles de millones de dólares)

\begin{tabular}{|l|c|c|c|c|c|c|}
\hline \multicolumn{4}{|c}{} & Estimación & \multicolumn{2}{c|}{ Pronóstico } \\
\hline & 2009 & 2010 & 2011 & 2012 & 2013 & 2014 \\
\hline Flujos de entrada & 674.2 & 1131.2 & 1018.5 & 818.1 & 994.8 & 1198.1 \\
\hline Flujos Privados & 593.7 & 1059.9 & 969.0 & 775.4 & 953.2 & 1152.1 \\
\hline Flujos Accionarios & 508.7 & 634.1 & 649.1 & 533.6 & 647.0 & 774.9 \\
\hline IED & 400.0 & 506.1 & 624.6 & 517.7 & 593.6 & 684.9 \\
\hline Flujos de Deuda & 85.0 & 425.8 & 339.9 & 241.8 & 306.2 & 377.2 \\
\hline Flujos Oficiales & 80.5 & 71.2 & 49.5 & 42.7 & 41.6 & 46.0 \\
\hline
\end{tabular}

Fuente: Global Economic Outlook, World Bank, Washington, June 2012

En el Cuadro 4, se aprecia la caída prevista por el BM, los flujos de entrada de capital hacia los países en desarrollo disminuyeron en 2012 en relación al año anterior. La mayor parte de esa disminución corresponde a los flujos de capital privado ${ }^{3}$ y de ello corresponde un alto porcentaje a los flujos de deuda. Para los años 2013 y 2014 los pronósticos indican una leve mejoría, encontrándose éstas todavía por debajo del pico de pre crisis (2007).

\section{Cuadro $\mathrm{N}^{\circ} 5$ \\ Flujos de Capital Privado según Regiones \\ (En porcentaje del PIB)}

\begin{tabular}{|l|c|c|c|c|}
\hline \multicolumn{1}{|c|}{ Regiones } & 2009 & 1010 & 2011 & 2012 \\
\hline Flujos Privados & 3.7 & 5.4 & 4.3 & 3.3 \\
\hline Este de Asia y Pacífico & 3.7 & 6.0 & 4.7 & 3.4 \\
\hline Europa y Asia Central & 2.7 & 5.0 & 3.6 & 2.0 \\
\hline América Latina y el Caribe & 3.9 & 6.0 & 4.8 & 4.1 \\
\hline Medio Oriente y Norte de África & 2.8 & 2.4 & 2.0 & 1.2 \\
\hline Sur de Asia & 4.6 & 5.0 & 3.9 & 3.3 \\
\hline África Subsahariana & 4.0 & 3.7 & 3.9 & 3.5 \\
\hline
\end{tabular}

Fuente: Global Economic Prospects, World Bank, Washington, January 2012.

En el Cuadro 5, según estimaciones del BM se registra, en términos relativos una desaceleración en las entradas de flujos de capital privado hacia las distintas regiones en desarrollo. Las regiones de Este de Asia y Pacífico y América Latina y el Caribe serían las más favorecidas por sus tasas de crecimiento del producto, aunque también se registran reducciones en 2012 en relación al año anterior.
El efecto que ha causado esta tendencia, las menores entradas de flujos de capital, ha sido una moderación en la acumulación de reservas internacionales por parte de los países en desarrollo durante 2012. Sin embargo, la continuación de la acumulación de reservas internacionales refleja la preocupación sobre la situación económica mundial y la sensación de que es necesario tomar medidas de precaución

3 Desde la crisis, los flujos internacionales de capital privado hacia los países en desarrollo se han mantenido extremadamente volátiles. A mediados de 2012, se desencadenaron nuevas inestabilidades asociadas a los temores de los inversionistas de cartera respecto a la sustentabilidad de las finanzas públicas en Europa, generando una huída hacia inversiones seguras (flight to safety). Asimismo, signos de desaceleración económica en Brasil, China e India redujeron los flujos hacia esos países. 
$\mathrm{y}$ autoseguro frente a la incertidumbre y posibles efectos externos.

El mayor nivel de reservas en monedas de los principales países desarrollados sobrepasa los flujos de capital, dando como resultado que los países en desarrollo y en transición continúan realizando transferencias netas de recursos financieros a los países desarrollados. En el 2012, estos flujos de salida de capital totalizaron aproximadamente $845 \mathrm{mil}$ millones de dólares, reduciéndose desde 1 billón de dólares en 2011.

\section{EL PANORAMA LATINOAMERICANO}

Según la CEPAL, tuvo que modificar sus estimaciones de crecimiento para la región para 2013, de 3.5 por ciento en Abril-13 a 3.0 por ciento en Junio-13. Esta baja en la estimación se debe a la desaceleración económica de Brasil y México y a las tendencias negativas en los últimos meses de países que venían creciendo a tasas elevadas como Chile, Perú y Panamá.

Debido a un escenario externo negativo, una alta dependencia de las exportaciones hacia Europa y China, un creciente aumento del déficit en la cuenta corriente de la balanza de pagos ${ }^{4}$, serias restricciones fiscales en América Central, México y el Caribe, y vulnerabilidad en América del Sur surgida a partir de su dependencia de recursos naturales. Estas debilidades podrían afectar el crecimiento en el corto y mediano plazo de la región.

La CEPAL, asimismo estima necesario una revisión de las previsiones de crecimiento mundial para 2013 (véase Cuadro 1) a un crecimiento similar al año 2012, debido a la continua recesión en la zona del euro durante el presente año. Se espera que los países en desarrollo sigan siendo los impulsores del crecimiento económico mundial, favorecidas por las políticas adoptadas por Estados Unidos y Japón para el repunte de estas economías y el mayor dinamismo económico mundial para 2014.

Por otra parte, dado que la actividad a nivel global se moderó más de lo esperado, incluso en las economías asiáticas, ello implicó una reducción del crecimiento de la demanda de bienes y servicios procedentes de América Latina y el Caribe. Junto a ello, se ha verificado una reducción en los precios de los commodities en los mercados internacionales y simultáneamente pérdidas en la relación de términos de intercambio de los países latinoamericanos y caribeños exportadores de productos básicos, los cuales dan cuenta de las tres cuartas partes de la producción total de la región. Por su parte, la demanda doméstica y la de inversiones, se restringió como resultado de las restricciones de política que se habían aplicado en varios países de la región.

En general, dada la alta dependencia de la región respecto a la evolución en los mercados internacionales de productos básicos, particularmente los países sudamericanos, los riesgos de mediano plazo que pudieran impactar esos mercados son de especial significado para esta subregión. Un crecimiento potencial menor también constituye un riesgo significativo desde una perspectiva intrarregional dado que el crecimiento económico de América Latina y el Caribe ha estado por encima de su tendencia histórica en los últimos diez años.

4 Que podría llegar al 2.0 por ciento del producto interno bruto (PIB) en 2013, el mayor desde el año 2011.

68/ QVIPURAMAYOC | Vol. 21(40) 2013 


\section{Cuadro $\mathrm{N}^{\circ} 6$ \\ Países que lideraran el crecimiento en América Latina en 2013}

(En variaciones porcentuales)

\begin{tabular}{|c|c|c|}
\hline Países & 2012 & $2013^{*}$ \\
\hline Paraguay & -1.8 & 12.5 \\
\hline Panamá & 10.5 & 7.5 \\
\hline Perú & 6.2 & 5.9 \\
\hline Bolivia & 5.0 & 5.5 \\
\hline Nicaragua & 4.0 & 5.0 \\
\hline Chile & 5.5 & 4.6 \\
\hline Argentina & 2.2 & 3.5 \\
\hline Brasil & 1.2 & 2.5 \\
\hline México & 3.8 & 2.8 \\
\hline
\end{tabular}

Fuente: Estudio Económico de América Latina y el Caribe 2013, CEPAL.

Brasil y Argentina representan el $40 \%$ del PIB Regional.

(2013*) Son estimaciones revisadas por CEPAL en Junio 2013

Estas nuevas previsiones $\left(2013^{*}\right)$, se sustentan en el desempeño económico durante el primer semestre de 2013, afectado por el descenso de los precios de varios productos de exportación de la región, especialmente de los minerales y metales, el petróleo y algunos alimentos, tendencia asociada a la recesión de la eurozona y a la desaceleración del crecimiento de la economía china.

\section{CONCLUSIONES}

Para los países de América Latina y el Caribe, los principales riesgos se vinculan con aquellos que afectan a la economía mundial. El principal riesgo a mediano plazo es que se produzca un escalamiento en la crisis de la zona del euro y que se concrete el llamado "abismo fiscal" en Estados Unidos. Sin embargo, la mayoría de los países desarrollados han adoptado una combinación de políticas de austeridad fiscal y de expansión monetaria destinadas a reducir la deuda pública y los costos de refinanciamiento de deuda para romper el círculo vicioso entre los problemas de deuda soberana y fragilidad en el sector bancario. Se espera que estas medidas calmen los mercados financieros y puedan restaurar la confianza en consumidores e inversionistas.
La incertidumbre debe ser enfrentada a través de cambios en la manera cómo se está enfrentando la crisis, en particular con una mayor consideración de los efectos que tienen las políticas nacionales a nivel internacional. En el caso de los países en desarrollo más grandes y que enfrentan serios riesgos de desaceleración, deben establecer políticas contracíclicas con mayor fuerza y que al mismo tiempo enfrenten los problemas estructurales de mediano plazo. Así China, tiene un amplio espacio de políticas para rebalancear su economía hacia un mayor peso de la demanda doméstica, incluyendo un mayor gasto del gobierno en servicios públicos como salud, educación y seguridad.

Como la economía mundial tiene hoy menos capacidad para enfrentar una nueva crisis como la de 2009, debido principalmente a que las economías industrializadas tienen un menor margen de acción: las tasas de interés están cercanas al cero por ciento en Estados Unidos y la Unión Europea, lo que se traduce en tasas de interés reales negativas, y por tanto, existen pocas posibilidades de introducir nuevos estímulos. Por lo que debería considerarse los crecientes desbalances en cuenta corriente a nivel internacional para corregir los desajustes económico a ese nivel, en

Vol. 21(40) 2013 | QVIPURAMAYOC /69 
cambio, se han priorizado las políticas para reducir los déficits, sin considerar el necesario estímulo a la demanda en las economías con excedentes externos (como Alemania, Holanda, China y Otras) que pudieran contribuir no sólo a una economía global más segura, sino también, a un probable mayor crecimiento en general.

Los precios de los alimentos han dejado atrás los elevados niveles recientes gracias a la mejora de las perspectivas de la oferta, pero se mantiene la escasez en los mercados debido a los niveles históricamente bajos de existencias. La probabilidad de fluctuaciones extremas de los precios en el plazo de nueve meses ha aumentado en el caso del maíz y el trigo desde octubre de 2012, lo cual indica que los riesgos al alza de los precios se han agudizado ligeramente.

Los precios de los metales, en general, han bajado desde comienzos de 2012 -tras la sólida reposición de las existencias en China y el marcado aumento de las existencias- debido al enfriamiento del consumo y la debilidad de la demanda de importaciones de China. Sin embargo, en el caso de algunos metales como el cobre, los precios se mantuvieron elevados porque la oferta continúa desfalleciente; que de otros, en los últimos tiempos pasaron a la sección superior de la curva de costos de la industria, de modo que los riesgos a la baja para los precios son mucho menores. Es importante considerar que la perspectiva de los precios de los metales están estrechamente vinculadas a la evolución del mercado chino, que consume más del 40 por ciento de todos los metales. El crecimiento de la demanda china se moderará a medida que la economía se oriente más a los servicios. Por lo que es de presumir un aumento de volatilidad en todos los mercados de materias primas.

Finalmente, el actual escenario pone de manifiesto problemas de sostenibilidad del crecimiento en la mayor parte de economías de la región y justifica plantear la necesidad de ampliar y diversificar sus fuentes de expansión.

\section{REFERENCIAS BIBLIOGRÁFICAS}

1. BARCENA, ALICIA, Presentación del Estudio Económico en América Latina y el Caribe 2013, elaborado por la Comisión Económica para América Latina, Santiago de Chile, 14 de Julio, 2013.

2. BANCO MUNDIAL, Perspectivas Económicas Mundiales, Washington, Junio, 2012.

3. BANCO MUNDIAL, Global Economic Prospects, Washington, January 2012.

4. CEPAL, Estudio Económico de América Latina y el Caribe 2013, Santiago de Chile, Julio, 2013.

5. CEPAL, Estudio Preliminar de las Economías de América Latina y el Caribe 2012, Santiago de Chile, Diciembre 2012.

6. FMI, Perspectivas de la Economía Mundial (WEO), Washington, Abril, 2013.

7. FMI, World Economic Outlook (WEO), Washington, October 2012.

8. FMI, Global Financial Stability Report, Washington, 2012.

9. HERRERA, BEATRIZ, La Crisis. Perspectiva del Empleo y el Comercio, Revista Quipukamayoc, Vol. 20 Enero-Junio, UNMSM, Lima, 2012.

10. OECD, Economic Outlook, Paris, September 2012.

11. OMC, Informe sobre el Comercio Mundial 2012, Ginebra, Julio, 2012.

12. SELA, El Actual Panorama Económico Mundial y sus Implicaciones para América Latina y el Caribe, Notas Estratégicas, Venezuela, Febrero, 2012.

13. WESP, World Economic Situation and Prospects as of mid-2013, United Nations, May 2013.

14. WESP, World Economic Situation and Prospects 2013, United Nations, January 2013.

15. WESP, World Economic Situation and Prospects 2012, United Nations. December 2012. 